\title{
Intra- and interlaboratory performance of antibiotic disk-diffusion-susceptibility testing of bacterial control strains of relevance for monitoring aquaculture environments
}

\author{
Geert Huys ${ }^{1, *}$, Margo Cnockaert ${ }^{1}$, Kerry Bartie $^{2}$, Dang Thi Hoang Oanh ${ }^{3}$, Nguyen \\ Thanh Phuong ${ }^{3}$, Temdoung Somsiri ${ }^{4}$, Supranee Chinabut ${ }^{4}$, Fatimah Md Yussoff ${ }^{5}$, \\ Mohamed Shariff ${ }^{5}$, Mauro Giacomini ${ }^{6}$, Stefania Bertone ${ }^{7}$, Jean Swings ${ }^{1,8}$, Alan Teale ${ }^{2}$ \\ ${ }^{1}$ Laboratory of Microbiology, Ghent University, K. L. Ledeganckstraat 35, 9000 Gent, Belgium \\ ${ }^{2}$ Institute of Aquaculture, University of Stirling, Stirling FK9 4LA, UK \\ ${ }^{3}$ Laboratory of Fish Diseases, College of Aquaculture and Fisheries, Can Tho University, Can Tho City, Vietnam \\ ${ }^{4}$ Aquatic Animal Health Research Institute, Kasetsart University Campus, Jatujak, Bangkok 10900, Thailand \\ ${ }^{5}$ Institute of Bioscience, Universiti Putra Malaysia, 43400 Serdang, Selangor, Malaysia \\ ${ }^{6}$ DIST, University of Genova, Via Opera Pia 13, 16145 Genova, Italy \\ ${ }^{7}$ RILAB stl, Via Guerrazzi 24/12B, 16146 Genova, Italy \\ ${ }^{8}$ BCCM/LMG Bacteria Collection, Ghent University, K. L. Ledeganckstraat 35, 9000 Gent, Belgium
}

\begin{abstract}
In the course of an international research project on hazard analysis of antimicrobial resistance in SE Asian aquaculture environments, 2 European Union and 3 SE Asian laboratories attempted to harmonize a procedure for antimicrobial agent susceptibility testing based on disk diffusion (DD). For this purpose, a selected panel of 10 bacterial control strains of relevance for monitoring warm-water aquaculture environments was sent by the central laboratory to the other participating laboratories. In each laboratory, 10 independently replicated DD determinations of each control strain to 6 antibiotics were performed using Iso-Sensitest Agar (ISA) according to a standard operating procedure (SOP); in total, this study thus yielded 300 data sets for all 5 laboratories. At the end of the study, strain authenticity of subcultures of the control strains used by the respective participating laboratories was verified by the central laboratory. Based on the arithmetic mean of 10 inhibition-zone diameter measurements and standard deviation (SD), intralaboratory SD variations ranged from 0 to $2 \mathrm{~mm}$ when $79 \%$ of the recorded data sets were considered. In $8 \%$ of the data sets, the SD value exceeded $4 \mathrm{~mm}$, which in most cases could be attributed to the fact that the data points for a given strain-disk combination were not normally distributed in one of the laboratories. At the interlaboratory level, $81 \%$ of the SD values based on global averaging of 50 data points per strain-disk combination were situated in the 0 to $5 \mathrm{~mm}$ range. Comparison with a minimal data set from literature of DD testing performed with Mueller-Hinton (MH) medium indicated that the use of either ISA or MH medium in DD testing has a limited impact on the method's precision among different laboratories. In conclusion, the current study has provided a validated SOP to promote the coordination and harmonization of DD-susceptibility methodologies for aquaculture-associated organisms at an international level. As one of the main action items for the future, new interpretive breakpoints should be specifically designed and validated for aquaculture drugs and organisms.
\end{abstract}

KEY WORDS: Antimicrobial agent susceptibility testing · Disk diffusion · Aquaculture monitoring · Intra- and interlaboratory harmonization - Standard operating procedure

Resale or republication not permitted without written consent of the publisher

\section{INTRODUCTION}

Despite the increase in strategies based on vaccination and probiotics, it is beyond doubt that antimicrobial agents remain important in controlling bacterial infections in many aquaculture regions worldwide. However, during the past 2 decades, it has become increasingly clear that many of these antibacterial agents have lost their effectiveness against several aquaculture pathogens. It is meanwhile widely ac- 
cepted that the uncontrolled and unrestricted use of these agents has certainly contributed to the selection and the widespread dissemination of antibiotic resistance in the aquaculture environment.

For the detection of newly emerging resistances and to evaluate the effectiveness of currently used drugs in aquaculture, long-term monitoring of susceptibility on a global scale is a key priority. Although there are various standardized methods available to perform such surveys, aquaculturists and microbiologists are often confronted with the fact that the existing knowledgebase consists mostly of data generated in a non-harmonized manner (Schnick 2001). Especially for fish pathogens and other aquaculture-associated organisms, it is not clear to what extent the use of different methods or multiple protocols describing a similar method affect the accuracy, precision and meaning of antimicrobial agent susceptibility testing (Smith 2001). In 2001, the participants in an international European Union (EU) workshop on minimum inhibitory concentration (MIC) methodologies in aquaculture put forward recommendations for uniform methods in the antimicrobial agent susceptibility testing of bacteria associated with fish diseases (Alderman \& Smith 2001). Despite the fact that provisional standardized protocols were designed for all major testing methods, including disk diffusion, agar dilution and broth dilution, it remains to be seen to what extent the proposed procedures will gain widespread acceptance. Moreover, one of the crucial factors in surveillance or monitoring of antimicrobial agent susceptibility, namely the evaluation of the external quality assurance, has so far been poorly addressed for organisms with relevance in monitoring aquaculture environments. Such international antimicrobial resistance surveillance systems already exist for clinical organisms, in order to identify the key problems leading to interpretation errors (Tenover et al. 2001). Recently, quality-control ranges for susceptibility testing of aquatic bacteria were proposed by the National Committee for Clinical Laboratory Standards (NCCLS) Veterinary Antimicrobial Susceptibility Testing-Aquaculture Working Group (VAST-AWG) using a limited selection of 2 control strains (Miller et al. 2003).

In the framework of an ongoing EU-funded project on hazard analysis of antimicrobial resistance in Southeast Asian (SEA) aquaculture environments (ASIARESIST; www.medinfo.dist.unige.it/Asiaresist/), 5 of the participating laboratories attempted to harmonize a procedure for disk-diffusion (DD)-susceptibility testing, with the ultimate aim of submitting locally obtained data to a single, central data collection. Essentially, the harmonization study comprised 3 phases. In the first phase, a selected panel of 10 control strains of relevance for monitoring warm-water aqua- culture environments was sent by the central laboratory to the other participating EU and SEA laboratories in which they were locally cryopreserved. The second phase consisted of 10 independently replicated DDsusceptibility tests of each control strain for 6 selected antibiotics following a standard operating procedure (SOP), after which data were submitted to the database maintained by the central laboratory. Finally, the participating laboratories were asked to send a subculture of each control strain back to the central laboratory for verification of strain authenticity. This approach allowed us to assess the intra- and interlaboratory proficiency and performance in an effort to coordinate and harmonize DD methodologies at an international level.

\section{MATERIALS AND METHODS}

Cultivation and distribution of bacterial strains. The selected panel of 10 bacterial control strains used in the harmonization study (Table 1) represented species that have been recognized as potential reservoir organisms of antibiotic resistance in freshwater or brackish water environments (Aeromonas hydrophila ssp. hydrophila, Acinetobacter junii, Escherichia coli, Salmonella enteritidis and Stenotrophomonas maltophilia) (Huys et al. 2000) or that have been primarily associated with diseases of certain aquaculture species (Aeromonas salmonicida ssp. salmonicida, Photobacterium damselae ssp. piscicida, Vibrio alginolyticus, V. anguillarum and Yersinia ruckeri). All strains were grown on IsoSensitest Agar (ISA; Oxoid, Basingstoke, UK) at $28^{\circ} \mathrm{C}$ to meet the optimum growth temperature of organisms isolated from or associated with warm-water species cultured in freshwater or brackish water systems, such as tilapia, sea bass, pangasius catfish and black tiger shrimp. For cultivation of halophilic organisms, ISA medium was supplemented with $2 \% \mathrm{NaCl}$ (Table 1).

In total, 5 laboratories participated in the harmonization study: (1) Laboratory of Microbiology, Ghent University, Ghent, Belgium; (2) Institute of Aquaculture,

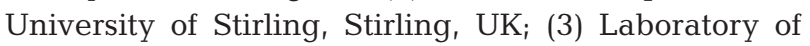
Fish Diseases, College of Aquaculture and Fisheries, Can Tho University, Can Tho, Vietnam; (4) Aquatic Animal Health Research Institute, Department of Fisheries, Kasetsart University Campus, Bangkok, Thailand; and (5) Faculty of Veterinary Medicine, Universiti Putra Malaysia, Selangor, Malaysia. Lyophilized subcultures of each control strain were maintained in the central laboratories in Ghent University and distributed as living cultures on small ISA slants to the other 4 participating laboratories. In each of these laboratories, control strains were checked for purity on ISA medium (with or without added $\mathrm{NaCl}$ ) and maintained on cryobeads (Microbank, Pro-Lab Diagnostics 
Table 1. Bacterial control strains used in this study. ATCC, American Type Culture Collection, Rockville, Maryland, USA; LMG, BCCM/LMG Bacteria Collection, Ghent University, Belgium; NCIMB, The National Collections of Industrial and Marine Bacteria, Aberdeen, UK

\begin{tabular}{|c|c|c|c|}
\hline Species & LMG no. & Other no. & $\begin{array}{l}\text { Growth } \\
\text { medium }\end{array}$ \\
\hline Aeromonas hydrophila ssp. hydrophila & LMG $2844^{\mathrm{a}}$ & ATCC 7966, NCIMB 86 & ISA \\
\hline Aeromonas salmonicida ssp. salmonicida & LMG $3780^{\mathrm{a}, \mathrm{b}}$ & ATCC 33658, NCIMB 1102 & ISA \\
\hline Acinetobacter junii & LMG 10577 & & ISA \\
\hline Escherichia coli & LMG $8223^{\mathrm{a}}$ & ATCC 25922, NCIMB 12210 & ISA \\
\hline Photobacterium damselae ssp. piscicida & LMG $7892^{\mathrm{a}, \mathrm{b}}$ & ATCC 33539, NCIMB 2184 & $\mathrm{ISA}+2 \% \mathrm{NaCl}$ \\
\hline Salmonella enteritidis & LMG $10395^{\mathrm{a}}$ & ATCC 13076 & ISA \\
\hline Stenotrophomonas maltophilia & LMG 11098 & & ISA \\
\hline Vibrio anguillarum & LMG $4437^{\mathrm{a}, \mathrm{b}}$ & ATCC 19264, NCIMB 6 & $\mathrm{ISA}+2 \% \mathrm{NaCl}$ \\
\hline Vibrio alginolyticus & LMG $4409^{\mathrm{a}}$ & ATCC 17749, NCIMB 1903 & $\mathrm{ISA}+2 \% \mathrm{NaCl}$ \\
\hline Yersinia ruckeri & LMG $3280^{\mathrm{a}, \mathrm{b}}$ & NCIMB 1316 & ISA \\
\hline
\end{tabular}

or Protect) at a minimal temperature of $-70^{\circ} \mathrm{C}$ during the entire harmonization study. Following the DD assay (see below), all participating laboratories were requested to send a living subculture of each control strain back to the central laboratory. In this laboratory, the cultures were checked for recovery and purity and subsequently subjected to GLC analysis of cellular fatty acid methyl esters (FAMEs) as described previously (Huys et al. 1994). The taxonomic authenticity of each subculture was checked by numerical comparison of its FAME profile with that of the original control strain supplied to the testing laboratories using Bionumerics version 3.0 software (Applied Maths, Belgium).

Disk-diffusion-susceptibility assay. Susceptibility testing with the DD method was performed according to a SOP that described the $3 \mathrm{~d}$ assay as agreed by all laboratories participating in the harmonization study. Upon subculturing the set of 10 control strains, each participating laboratory performed the antibiotic DD assay 10 times during independent trials.

On Day 1, each control strain was cultured on ISA medium (supplemented with $2 \% \mathrm{NaCl}$ if required) at $28^{\circ} \mathrm{C}$ for $24 \mathrm{~h}$, under aerobic conditions starting from the cryopreserved subculture. Cryobeads were streaked out on ISA plates in order to obtain distinct colonies. During the same day, the desired volume of ISA medium for DD testing was prepared according to the manufacturer's (Oxoid) instructions. ISA plates were poured onto a flat, horizontal surface, each to an identical depth of $5 \pm 1 \mathrm{~mm}$ (corresponding to $20 \pm 1 \mathrm{ml}$ of medium in petri dishes of $10 \mathrm{~cm}$ radius). In addition, a sufficient number of tubes containing $5 \mathrm{ml}$ sterile physiological saline (PS; double-distilled water containing $0.85 \% \mathrm{NaCl}$ ) was prepared.

On Day 2, a number of colonies with similar morphology were harvested from the freshly grown plate culture to prepare a suspension in $5 \mathrm{ml}$ PS with a tur- bidity corresponding to 1.0 of the McFarland standard (i.e. $3 \times 10^{8} \mathrm{CFU} \mathrm{ml}{ }^{-1}$ ). Using a micropipette, $100 \mu \mathrm{l}$ of the standardized suspension was spotted onto the surface of an ISA plate, after which the suspension drop was spread plated using a sterile glass triangle rod to obtain semi-confluent growth. The plates were allowed to dry for a maximum of $15 \mathrm{~min}$. Using an Oxoid disk dispenser, disks (diameter: $6 \mathrm{~mm}$ ) of 6 different antibiotics were applied onto the agar surface, i.e. tetracycline (TE30), oxytetracycline (OT30), streptomycin (S25), chloramphenicol (C30), ampicillin (AMP25) and oxolinic acid (OA2). The plates were incubated at $28^{\circ} \mathrm{C}$ for $24 \mathrm{~h}$ under aerobic conditions.

On Day 3, the diameter of the inhibition zone (if present) around each disk was measured to the nearest millimeter from the point of abrupt inhibition of growth, using callipers or a millimeter ruler. Where there was any doubt, the point of $80 \%$ inhibition was considered as the zero edge. Plates on which control strains produced isolated colonies or heavy growth instead of semi-confluent growth were not read. If zones of inhibition produced by adjacent disks overlapped in such a way that 2 measurements at right angles could not be made, the zones around these disks were not recorded. This problem could usually be solved by adapting the order of the disks in the dispenser system or by using 2 plates each containing 3 disks positioned at maximal distances. Finally, it was agreed that zones demonstrating significant distortion from circular should not be reported.

Data processing and statistical analysis. For each participating laboratory, results of 10 independently replicated DD test series were collected in a central database, after which a normality control of the distribution of the 10 replicate values per strain-disk combination was performed. For this control, a model of normal statistical distribution was implemented for each 
data set by applying the statistical parameters of the specific set. Based on a threshold level chosen with the Otsu method (Otsu 1979), a given set was defined as normally or non-normally distributed. For each partner laboratory, a maximal number of 6 non-normalized data sets, corresponding to $10 \%$ of the total number of strain-disk combinations $(\mathrm{n}=60 ; 10$ strains tested for 6 disks) recorded per partner, were allowed.

Evaluation of the intralaboratory performance for each participant was based on the arithmetic mean (AM) of 10 independent replicate determinations and the resulting standard deviation (SD). The statistical variance at the intralaboratory level for each strain-disk combination was expressed by the coefficient of variation $(\% \mathrm{CV}=[\mathrm{SD} / \mathrm{AM}] \times 100)$. Intralaboratory variances were considered to be statistically acceptable at \%CV values of $\leq 20 \%$.

The interlaboratory performance was assessed by calculation of the overall arithmetic mean from the 50 data points of each strain-disk combination and the resulting SD. Based on combined use of ANOVA and the Duncan method, clusters of similarities among the 5 laboratories were calculated. Strain-disk data sets that grouped in clusters containing 3 or more laboratories were considered to display significant interlaboratory similarity.

\section{RESULTS}

\section{Verification of strain authenticity}

Subsequent to the 10 independently replicated DD assays according to the SOP, the central laboratory received fresh subcultures of the control strains used by the respective participating laboratories. Upon receipt, subcultures were checked for recovery and purity on ISA medium. In one instance, one of the received subcultures of Stenotrophomonas maltophilia LMG 11098 yielded multiple colony types. Therefore, a second subculture was requested from the laboratory concerned. In other cases, subcultures of Photobacterium damselae ssp. piscicida and Aeromonas salmonicida ssp. salmonicida received from 2 SEA participants proved difficult to recover, and several batches of new subcultures had to be sent until recovery was successful. The taxonomic authenticity of pure subcultures was checked by GLC-FAME analysis. Numerical comparison with the fatty acid profiles of the original lyophilized cultures indicated that all received subcultures were assigned to the correct species (data not shown).

\section{Normality of the data sets}

Normality control of the 300 data sets (i.e. 60 strain-disk combinations recorded in Laboratories 1 to $5)$ indicated that only 16 sets (5\%) displayed a nonnormal distribution. In 2 of the laboratories, all 60 data sets were normally distributed (Table 2). The number of non-normal sets in the other laboratories ranged from 4 to 7 (Table 2), which is acceptable based on the $10 \%$ threshold of the non-normally distributed data sets allowed per laboratory.

\section{Intralaboratory performance}

From the 300 recorded data sets, 92\% were situated in the $\% \mathrm{CV}$ range of $0 \%$ to $20 \%$, indicating that these data sets all share a statistically acceptable intra-assay variance. Of the remaining 24 data sets that displayed $\mathrm{CV}$ values of $>20 \%$, 21 were confined to Laboratories 3 and 5 (Table 2). In these 2 laboratories, 5 out of the 6 data sets recorded for Stenotrophomonas maltophilia LMG 11098 and Photobacterium damselae ssp. piscicida LMG 7892, respectively, exhibited an intra-assay variance exceeding $20 \% \mathrm{CV}$.

As shown in Table 3, the differences among the intralaboratory AM values ranged from 0 to $17 \mathrm{~mm}$ within 1 specific strain-disk combination. The performances within each laboratory were evaluated on the basis of the SD calculated from the intralaboratory average of 10 independently replicated zone diameters. The majority of these SD values (79\%) were in the range of 0 to $2 \mathrm{~mm}$, and only for $8 \%$ of the 300 recorded data sets did the $\mathrm{SD}$ value exceed $4 \mathrm{~mm}$ (Table 3, Fig. 1). Many of the latter cases could be attributed to the fact that the data points for a given strain-disk combination were not normally distributed in one of the laboratories. As a consequence, this one laboratory often reported a mean value that was clearly outside the range of means recorded by the other laboratories. For instance, in the case of AMP 25 disk testing with Photobacterium damselae ssp. pisci-

Table 2. Normality and intra-assay parameter intervals of the data sets recorded by 5 laboratories. Values are number of data sets per laboratory

\begin{tabular}{|lrrrrrc|}
\hline & Lab 1 & Lab 2 & Lab 3 & Lab 4 & Lab 5 & Total (\%) \\
\hline $\begin{array}{l}\text { Normality test } \\
\text { Normal distribution }\end{array}$ & 60 & 60 & 53 & 55 & 56 & $284(95)$ \\
Non-normal distribution & 0 & 0 & 7 & 5 & 4 & $16(5)$ \\
$\%$ CV intervals & & & & & & \\
$0-5 \%$ & 18 & 40 & 24 & 17 & 7 & $106(35)$ \\
$5-10 \%$ & 26 & 17 & 16 & 31 & 27 & $117(39)$ \\
$10-20 \%$ & 15 & 3 & 10 & 10 & 15 & $53(18)$ \\
$>20 \%$ & 1 & 0 & 10 & 2 & 11 & $24(8)$ \\
\hline
\end{tabular}


cida LMG 7892, the SD value of 4 of the 5 participating laboratories fell in the range of 1 to $5 \mathrm{~mm}$, whereas the fifth laboratory generated an SD value of $19 \mathrm{~mm}$ (Table 3).

\section{Interlaboratory performance}

Based on the calculation of similarity clusters, 44 of the 60 strain-disks combinations $(73 \%)$ were found to belong to clusters containing 3 or more laboratories (Fig. 2) and thus displayed significant interlaboratory similarity. The remaining combinations belonged to distinct or overlapping clusters containing 2 laboratories or did not make up part of any cluster due to the fact that interlaboratory dissimilarities were too high.

At the interlaboratory level, $81 \%$ of the SD values based on the global averaging of 50 data points per strain-disk combination were situated in the 0 to $5 \mathrm{~mm}$ range (Fig. 3). Of all SD values, $53 \%$ fell inside the 0 to $3 \mathrm{~mm}$ range. Overall, no particular association between high SD values ( $>5 \mathrm{~mm}$ ) and specific disks could be observed. However, it is noteworthy that the interlaboratory SD values obtained for the 6 disks tested with the control strains Yersinia ruckeri LMG 3280 and Photobacterium damselae ssp. piscicida LMG 7892 all ranged relatively high, from 4 to $9 \mathrm{~mm}$ (Table 3 ).

\section{DISCUSSION}

Assessment of the accuracy and meaning of antimicrobial DD-susceptibility testing in which data are determined and centrally submitted by different laboratories at an international level is a stepwise process in which various aspects of quality control and proficiency testing should be considered. Once a SOP for DD testing is formulated, it is of utmost importance that its performance is evaluated individually within each of the participating laboratories. For each strain-disk combination included in the present study, 10 independently replicated DD assays were performed per laboratory for the purpose of statistical analysis at the intralaboratory level. The number of non-normally distributed data sets per laboratory did not exceed the acceptable limit of $10 \%$ required for statistical analysis, thus indicating that data sets used for the intraassay evaluation generally displayed a normal distribution. Furthermore, 82 to $98 \%$ of the recorded data sets per laboratory showed an acceptable intra-assay variance of $\leq 20 \%$ (Table 2 ). At the level of the intralaboratory SD values, this was reflected by a narrow SD range of 0 to $2 \mathrm{~mm}$ when $79 \%$ of the 300 data sets recorded by all laboratories were considered. Most of the SD values that exceeded $2 \mathrm{~mm}$ were limited to spe-
Table 3. Intralaboratory average ranges, interlaboratory averages and standard deviations of disk-diffusion data for 60 strain-disk combinations (AMP, ampicillin; $\mathrm{C}$, chloramphenicol; OA, oxolinic acid; OT, oxytetracycline; $\mathrm{S}$, streptomycin; $\mathrm{TE}$, tetracycline)

\begin{tabular}{|c|c|c|c|c|c|}
\hline \multirow[t]{2}{*}{ Control strain } & \multirow{2}{*}{$\begin{array}{l}\text { Antibiotic } \\
\text { disk }\end{array}$} & \multicolumn{2}{|c|}{ Intralaboratory } & \multicolumn{2}{|c|}{ Interlaboratory } \\
\hline & & Range & SD & Average & $\mathrm{SD}$ \\
\hline \multirow{6}{*}{$\begin{array}{l}\text { Escherichia coli } \\
\text { LMG } 8223\end{array}$} & $\mathrm{~S} 25$ & $15-21$ & $1-2$ & 18 & 2 \\
\hline & TE30 & $23-31$ & $1-2$ & 26 & 3 \\
\hline & C30 & $23-29$ & $1-3$ & 25 & 3 \\
\hline & ОТ30 & $23-31$ & $1-2$ & 27 & 3 \\
\hline & AMP25 & $17-24$ & $1-3$ & 21 & 3 \\
\hline & OA2 & $22-28$ & $1-2$ & 24 & 2 \\
\hline \multirow{6}{*}{$\begin{array}{l}\text { Stenotrophomonas } \\
\text { maltophilia } \\
\text { LMG } 11098\end{array}$} & $\mathrm{~S} 25$ & $7-18$ & $1-7$ & 11 & 5 \\
\hline & TE30 & $9-22$ & $1-5$ & 17 & 5 \\
\hline & C30 & $12-20$ & $1-2$ & 16 & 3 \\
\hline & OT30 & $9-22$ & $1-4$ & 16 & 5 \\
\hline & AMP25 & $9-26$ & $1-5$ & 18 & 7 \\
\hline & OA2 & $7-14$ & $1-3$ & 11 & 3 \\
\hline \multirow{6}{*}{$\begin{array}{l}\text { Acinetobacter junii } \\
\text { LMG } 10577\end{array}$} & $\mathrm{~S} 25$ & $20-27$ & $1-3$ & 23 & 3 \\
\hline & TE30 & $22-36$ & $1-2$ & 28 & 5 \\
\hline & C30 & $14-26$ & $1-3$ & 20 & 4 \\
\hline & OT30 & $22-34$ & $1-2$ & 27 & 5 \\
\hline & AMP25 & $22-33$ & $1-2$ & 26 & 5 \\
\hline & OA2 & $19-25$ & $1-2$ & 21 & 3 \\
\hline \multirow{6}{*}{$\begin{array}{l}\text { Salmonella } \\
\text { enteritidis } \\
\text { LMG } 10395\end{array}$} & S25 & $15-21$ & 1 & 18 & 2 \\
\hline & TE30 & $25-31$ & $1-2$ & 27 & 3 \\
\hline & C30 & $22-31$ & $1-2$ & 26 & 3 \\
\hline & ОТ30 & $24-32$ & $1-2$ & 27 & 3 \\
\hline & AMP25 & $28-34$ & $1-2$ & 30 & 3 \\
\hline & OA2 & $19-25$ & $0-4$ & 22 & 3 \\
\hline \multirow{6}{*}{$\begin{array}{l}\text { Aeromonas } \\
\text { hydrophila } \\
\text { subsp. hydrophila } \\
\text { LMG } 2844\end{array}$} & S25 & $18-25$ & $1-2$ & 22 & 2 \\
\hline & TE30 & $28-33$ & $1-4$ & 29 & 3 \\
\hline & C30 & $30-37$ & $1-4$ & 32 & 3 \\
\hline & ОТ30 & $26-34$ & $1-3$ & 29 & 3 \\
\hline & AMP25 & 6 & 0 & 6 & 0 \\
\hline & OA2 & $24-31$ & $1-3$ & 27 & 3 \\
\hline \multirow{6}{*}{$\begin{array}{l}\text { Aeromonas } \\
\text { salmonicida } \\
\text { subsp. salmonicida } \\
\text { LMG } 3780\end{array}$} & S25 & $17-18$ & $1-4$ & 17 & 2 \\
\hline & TE30 & $29-37$ & $1-4$ & 32 & 4 \\
\hline & C30 & $31-39$ & $1-2$ & 34 & 3 \\
\hline & OT30 & $29-37$ & $1-3$ & 33 & 3 \\
\hline & AMP25 & $34-42$ & $1-5$ & 37 & 5 \\
\hline & OA2 & $26-35$ & $1-4$ & 31 & 3 \\
\hline \multirow{6}{*}{$\begin{array}{l}\text { Yersinia ruckeri } \\
\text { LMG } 3280\end{array}$} & S25 & $19-27$ & $2-4$ & 21 & 4 \\
\hline & TE30 & $30-46$ & $1-4$ & 37 & 6 \\
\hline & C30 & $25-41$ & $1-4$ & 33 & 6 \\
\hline & OT30 & $30-46$ & $1-4$ & 37 & 6 \\
\hline & AMP25 & $26-41$ & $2-7$ & 32 & 7 \\
\hline & OA2 & $24-38$ & $1-7$ & 30 & 6 \\
\hline \multirow{6}{*}{$\begin{array}{l}\text { Vibrio alginolyticus } \\
\text { LMG } 4409\end{array}$} & $\mathrm{~S} 25$ & $11-19$ & $1-5$ & 15 & 4 \\
\hline & TE30 & $24-33$ & $2-7$ & 27 & 6 \\
\hline & C30 & $27-38$ & $2-5$ & 32 & 5 \\
\hline & OT30 & $25-34$ & $2-6$ & 29 & 5 \\
\hline & AMP25 & $6-11$ & $0-3$ & 7 & 2 \\
\hline & OA2 & $19-27$ & $1-6$ & 23 & 5 \\
\hline \multirow{6}{*}{$\begin{array}{l}\text { Photobacterium } \\
\text { damselae } \\
\text { subsp. piscicida } \\
\text { LMG } 7892\end{array}$} & S25 & $10-19$ & $1-6$ & 14 & 5 \\
\hline & TE30 & $28-38$ & $1-8$ & 31 & 6 \\
\hline & C30 & $25-40$ & $1-7$ & 32 & 7 \\
\hline & OT30 & $27-39$ & $1-8$ & 32 & 6 \\
\hline & AMP25 & $18-28$ & $1-19$ & 21 & 9 \\
\hline & OA2 & $18-30$ & $1-3$ & 26 & 5 \\
\hline \multirow{6}{*}{$\begin{array}{l}\text { Vibrio anguillarum } \\
\text { LMG } 4437\end{array}$} & S25 & $10-18$ & $1-4$ & 13 & 4 \\
\hline & TE30 & $29-37$ & $1-3$ & 33 & 3 \\
\hline & C30 & $30-38$ & $1-2$ & 34 & 3 \\
\hline & OT30 & $30-38$ & $1-3$ & 34 & 3 \\
\hline & AMP25 & $12-17$ & $1-3$ & 15 & 2 \\
\hline & OA2 & $29-34$ & $1-2$ & 31 & 3 \\
\hline
\end{tabular}




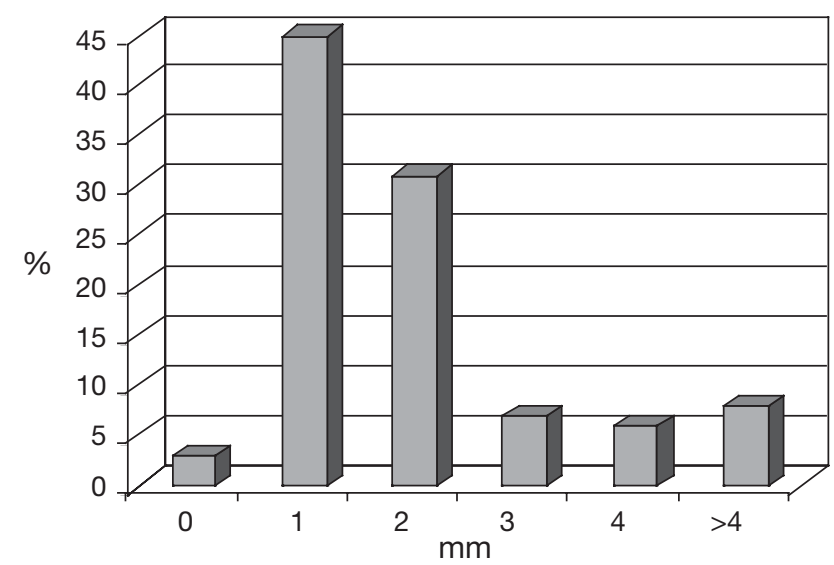

Fig. 1. Distribution of intralaboratory standard deviations of 300 data sets encompassing 60 strain-disk combinations analyzed in 5 laboratories during 10 independently replicated assays

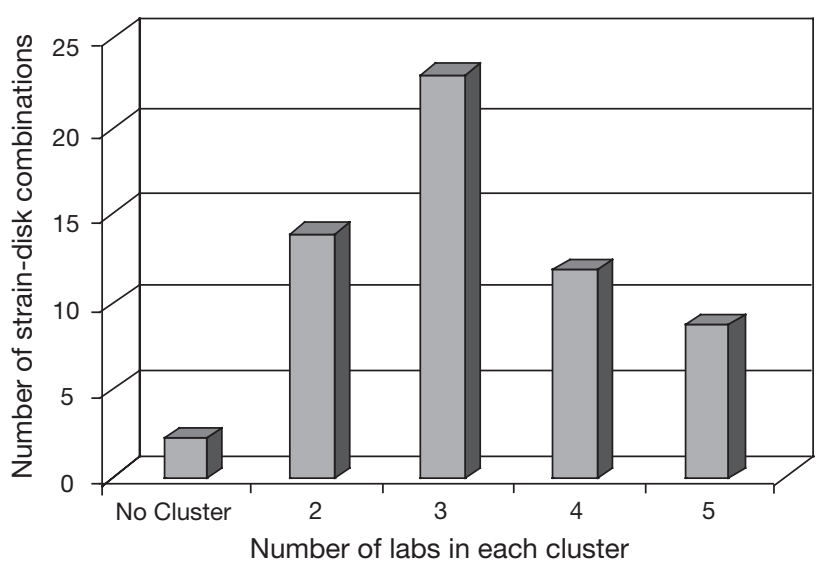

Fig. 2. Distribution of ANOVA-Duncan similarity clusters among 60 strain-disk combinations analyzed in 5 laboratories during 10 independently replicated assays

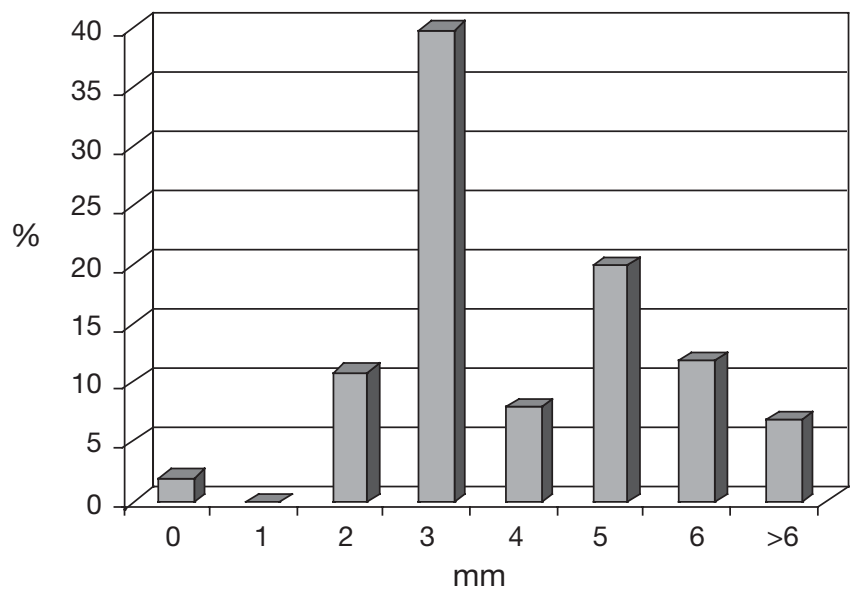

Fig. 3. Distribution of interlaboratory standard deviations of 300 data sets encompassing 60 strain-disk combinations analyzed in 5 laboratories during 10 independently replicated assays cific strain-disk combinations where the zone diameter of 1 of the laboratories was out of range compared to the values of the other 4 laboratories due to a nonnormal distribution profile of the data from that one laboratory. Comparison of \% CV values revealed that cases of unacceptable intra-assay variance were not randomly distributed among the 5 laboratories, but mainly occurred in the data sets of Laboratories 3 and 5. Given the fact that no special attention was paid to the possible effects of lot-to-lot variations of ISA medium and antibiotic disks, a maximum zone diameter variation of $2 \mathrm{~mm}$ can be regarded as an acceptable tolerance limit for further studies to evaluate the intralaboratory performance of DD-susceptibility testing on a larger scale.

For the purpose of harmonizing the interlaboratory logistics at the level of quality assurance and control, 3 main issues should be carefully evaluated in the design of such a harmonization study: (1) the method of choice, (2) the type and concentration of antimicrobial agents in the test panel and (3) the panel of control strains. The SOP used in this study for DD testing of aquatic bacteria was mainly inspired by the British Society for Antimicrobial Chemotherapy (BSAC) system, which relies on the use of ISA medium (Andrews et al. 2001). The selection of ISA was based on our good experience with this medium for culturing aquatic bacteria and on the fact that it consists of a standardized formula that does not require adjustment of ionic concentrations to minimize possible antagonistic effects. In contrast, Alderman \& Smith (2001) suggested that the introduction of a new protocol for susceptibility testing of bacteria associated with fish diseases may only gain widespread acceptance when based on the more widely adopted NCCLS protocol that makes use of Mueller-Hinton (MH) agar. Despite the fact that both methodologies are generally considered to be only partly equivalent, a study of Koeth et al. (2000) highlighted that Iso-Sensitest Broth is an acceptable alternative to cation-adjusted $\mathrm{MH}$ medium in MIC testing. Using ISA medium, the results of our harmonization study indicated that $73 \%$ of the investigated strain-disk combinations belonged to an ANOVA-Duncan similarity cluster comprising 3 or more laboratories, which indicates that these combinations display acceptable interlaboratory similarity. This level of compatibility was translated into an overall interlaboratory SD range of 0 to $5 \mathrm{~mm}$ when the scores of $81 \%$ of the investigated strain-disk combinations were considered (Fig. 3). In comparison, results of a study performed by the NCCLS Subcommittee VASTAWG using $\mathrm{MH}$ agar, in which 10 laboratories participated, indicated that the variation in the interlaboratory range determined following 24 to $28 \mathrm{~h}$ of incubation was 8 to $20 \mathrm{~mm}$ in DD testing of 2 control 
strains at $28^{\circ} \mathrm{C}$ (Miller et al. 2003). However, it should be stressed that the number of data points per strain-disk combination reported by Miller et al. was much higher $(295 \leq \mathrm{n} \geq 540)$ than generated in the present study $(\mathrm{n}=50)$. Comparison of the interlaboratory ranges for OT30 and OA2 DD testing using the control strain Escherichia coli ATCC 25922 between the present study (21 to $33 \mathrm{~mm}$ and 20 to $29 \mathrm{~mm}$, respectively) (data not shown) and those obtained by the VASTAWG (22 to $30 \mathrm{~mm}$ and 24 to $35 \mathrm{~mm}$, respectively) (Miller et al. 2003) indicate a moderate to excellent degree of agreement between studies. Likewise, the interlaboratory ranges for OT30 and OA2 DD testing at $28^{\circ} \mathrm{C}$ of the control strain Aeromonas salmonicida ssp. salmonicida ATCC 33658 obtained by us (25 to $39 \mathrm{~mm}$ and 20 to $38 \mathrm{~mm}$, respectively [data not shown]) and by the VAST-AWG (26 to $37 \mathrm{~mm}$ and 30 to $43 \mathrm{~mm}$, respectively) can be considered compatible. Although very preliminary, these comparisons seem to indicate that the use of either ISA or MH agar in DD testing has a limited impact on the method's precision among different laboratories.

The panel of antimicrobial agents tested in the present study represent different classes of agents such as tetracyclines (tetracycline and oxytetracycline), $\beta$-lactams (ampicillin), aminoglycosides (streptomycin), quinolones (oxolinic acid) and chloramphenicol. The differences in the structural properties and inhibition spectra of these agents allowed verification of the performance of a SOP for DD-susceptibility testing on a taxonomically diverse set of control strains of aquaculture-associated organisms across different laboratories. Obviously, this panel should be further extended with agents that are of specific relevance to the aquacultural environment, including drugs that are approved for use and those that are currently banned, but still used illegally in certain regions. In this context, resistance to florfenicol, nitrofurantoin, norfloxacin and trimethoprim-sulfamethoxazole should be considered in future monitoring studies.

Although often neglected, the interlaboratory circulation of a set of taxonomically well-characterized control strains in harmonization surveys of DD-susceptibility testing should ideally be accompanied by a control of their taxonomic authenticity. In the course of the present study, occasional problems with the purity or recovery of the control strains were noted upon re-submission of the working cultures to the central laboratory. Clearly, this finding highlights the necessity for a coordinated strainverification procedure prior to central data submission and processing. For antimicrobial susceptibility testing of bacteria associated with fish diseases, Alderman \& Smith (2001) proposed a panel of 4 control strains, including Aeromonas salmonicida ssp. salmonicida NCIMB 1102, Photobacterium damsela ssp. piscida NCIMB 2058, Vib- rio anguillarum NCIMB 6 and Yersinia ruckeri NCIMB 2194, based on their assumed susceptibility to a broad range of antimicrobial agents. Recently, the VAST-AWG (Miller et al. 2003) recommended classification of fishassociated organisms for the purpose of standardized DD-susceptibility testing into 5 groups, i.e. Group 1 (including Enterobacteriaceae, aeromonads and nonobligate halophilic vibrios), Group 2 (including obligate halophilic vibrios and Photobacterium spp.), Group 3 (including flavobacteria), Group 4 (including lactic acid bacteria) and Group 5 (including psychrophilic $A$. salmonicida, V. salmonicida and Gram-positive rods). Because the ASIARESIST project envisages resistance monitoring in mesophilic heterotrophs isolated from or associated with warm-water aquaculture species, the control strains used in the present study primarily encompassed VAST-AWG Groups 1 and 2, because these organisms grow well on standardized media such as ISA or $\mathrm{MH}$ agar at $28^{\circ} \mathrm{C}$, keeping in mind that members of Group 2 ( $V$. anguillarum, $V$. alginolyticus and $P$. damselae ssp. piscicida) require medium supplementation with $1.5 \%$ to $2.0 \% \mathrm{NaCl}$. As clearly shown for control strain $Y$, ruckeri LMG 3280, many of the strain-disk combinations for which the interlaboratory $\mathrm{SD}$ was $\geq 6 \mathrm{~mm}$ were associated with large inhibitionzone diameters $(\geq 25 \mathrm{~mm}$ ) read from plates with translucent growth (Table 3). This finding may reflect the fact that this organism was not tested under its optimal growth conditions. In addition, it is clear that the size of the inhibition zones displayed by any control strain may influence the reproducibility, and thus the performance of interpretive antibiogram reading among the different laboratories. Hence, the interlaboratory SD is expected to be much lower when smaller zone diameters are to be compared. In future selections of new control strains for DD-susceptibility testing of aquatic organisms, it can therefore be recommended to also include strains exhibiting multiple high-level or intermediate resistance phenotypes, which can be easily recognized by laboratories worldwide.

In his conclusion with respect to the validity and meaning of any susceptibility-testing method, Smith (2001) suggested that 3 main issues should be carefully considered. The first 2, i.e. the formulation of a SOP and the validation of its application, have been addressed in the reported study through the definition of a well-defined procedure for evaluating the intra- and interlaboratory proficiency and performance of the DD-susceptibility testing of aquatic and aquacultureassociated bacteria. The tolerance limits and potential bottlenecks that were observed in this harmonization survey may assist other workers to perform future resistance-monitoring studies in specific aquaculture settings. The third issue in Smith's conclusion, being the global establishment of susceptibility breakpoints 
for aquaculture drugs and organisms towards the clinical interpretation of data generated by the use of a SOP, clearly is still in its infancy. Next to the worldwide validation, acceptance and dissemination of a given DD methodology, the effects of growth temperature, nutritional requirements and salinity on the definition of breakpoints have not been properly investigated for many aquatic and aquaculture-associated organisms.

Acknowledgements. This work was carried out in the framework of EU-INCO-DEV Contract ICA4-CT-2001-10028 of the European Commission. G.H. is a postdoctoral fellow of the Fund for Scientific Research-Flanders (Belgium) (F.W.O.Vlaanderen).

\section{LITERATURE CITED}

Alderman DJ, Smith P (2001) Development of draft protocols of standard reference methods for antimicrobial agent susceptibility testing of bacteria associated with fish diseases. Aquaculture 196:211-243

Andrews JM, for the BSAC Working Party on Susceptibility Testing (2001) BSAC standardized disc susceptibility testing method. J Antimicrob Chemother 48[Suppl S1]: 43-57

Huys G, Vancanneyt M, Coopman R, Janssen P, Falsen E, Altwegg M, Kersters K (1994) Cellular fatty acid composition as a chemotaxonomic marker for the differentiation of phenospecies and hybridization groups in the genus

Editorial responsibility: David W. Bruno,

Aberdeen, UK
Aeromonas. Int J Syst Bacteriol 44:651-658

Huys G, Rhodes G, McGann P, Denys R, Pickup R, Hiney M, Smith P, Swings J (2000) Characterization of oxytetracycline-resistant heterotrophic bacteria originating from hospital and freshwater fishfarm environments in England and Ireland. Syst Appl Microbiol 23:599-606

Koeth LM, King A, Knight H, May J, Miller LA, Phillips I, Poupard JA (2000) Comparison of cation-adjusted Mueller-Hinton broth with Iso-Sensitest broth for the NCCLS broth microdilution method. J Antimicrob Chemother 46:369-376

Miller RA, Walker RD, Baya A, Clemens K and 8 others (2003) Antimicrobial susceptibility testing of aquatic bacteria: quality control disk diffusion ranges for Escherichia coli ATCC 25922 and Aeromonas salmonicida subsp. salmonicida ATCC 33658 at 22 and $28^{\circ} \mathrm{C}$. J Clin Microbiol 41: $4318-4323$

Otsu N (1979) A threshold selection method for grey-level histogram. IEEE (Inst Electro Electron Eng) Trans Syst Man Cybernatics 9:62-66

Schnick RA (2001) International harmonization of antimicrobial sensitivity determination for aquaculture drugs. Aquaculture 196:277-288

Smith P (2001) Accuracy, precision and meaning of antimicrobial agent susceptibility testing of bacteria associated with fish diseases. Aquaculture 196:253-266

Tenover FC, Mohammed MJ, Stelling J, O'Brien T, Williams R (2001) Ability of laboratories to detect emerging antimicrobial resistance: proficiency testing and quality control results from the World Health Organization's external quality assurance system for antimicrobial susceptibility testing. J Clin Microbiol 39:241-250

Submitted: January 8, 2004; Accepted: April 8, 2005

Proofs received from author(s): September 16, 2005 